$(63.3 \%)$ attended regular classes, $11(18.3 \%)$ had ADHD and $12(20 \%)$ had a learning disability at onset. After initiating antiepileptic medication, mean follow-up was 53 months (range 24-128 months). Seizures were completely controlled for at least 24 months in 29 $(48.3 \%)$, patients were seizure free for $>12$ months in 44 (73.3\%), seizures recurred within 12 months in $16(26.7 \%)$, and were intractable in $4(6.7 \%)$. Outcome was poor in $8(13.3 \%)$ and very poor in $4(6.7 \%)$. Risk factors for a poor outcome were a seizure recurrence within $6-12$ months after initiating therapy $(\mathrm{p}=0.006)$, and developmental delay at onset $(\mathrm{p}=0.023)$. (Tang-Wai R, Oskoui M, Webster R, Shevell M. Outcomes in pediatric epilepsy: seeing through the fog. Pediatr Neurol October 2005;33:244-250). (Respond: Dr Shevell, Room A514, Montreal Children's Hospital, 2300 Tupper Street, Montreal, Quebec H3H 1P3, Canada).

COMMENT. Cryptogenic epilepsy refers to seizures without genetic or known cause but presumed to be symptomatic, possibly a subtle cerebral dysgenesis undetected by MRI. The term idiopathic is reserved for seizures solely genetic in origin. Cryptogenic epilepsy in childhood generally has a favorable outcome, approximately $50 \%$ being seizure-free for more than 2 years after starting AEDs. Risk factors for a poor outcome include seizure recurrence within 6-12 months of treatment initiation and developmental delay at seizure onset. As expected, seizure intractability and poor outcome are correlated with the need for addition of a second AED. Except for developmental delay, initial clinical features are not helpful in predicting outcome. Despite the frequency of ADHD and learning disabilities, more than half the patients do not require special classes. Normal neurologic examination and the absence of comorbid disorders at onset of seizures do not always correlate with a favorable outcome, $55 \%$ requiring more than one AED. Seizure recurrences and developmental delay indicate a need for aggressive therapy.

\title{
COGNITIVE DEFICITS RELATED TO FOCAL SPIKE LOCATION IN BENIGN PARTIAL EPILEPSY
}

The topographic relation between focal spikes and neuropsychological deficits in children with benign partial epilepsy (BPE) was investigated using magnetoencephalography (MEG), electroencephalography (EEG) and MRI in a study of 27 children at University Children's Hospital, Tuebingen, Germany, and University of Trento, Italy. Location of spikes was determined by dipole source estimation. Of 20 children with sufficient MEG data, 13 showed focal spikes in the perisylvian region, and 7 in the occipital region. Five had bilateral or multiple foci. Left perisylvian spikes correlated with significantly lower language test scores $(\mathrm{p}=0.01)$, and occipital spikes with lower scores in simultaneous information processing $(\mathrm{p}=0.01)$, especially in visual transformation tasks. Focal interictal spikes may interfere with complex cognitive functions. (Wolff M, Weiskopf N, Serra E et al. Benign partial epilepsy in childhood: selective cognitive deficits are related to the location of focal spikes determined by combined EEG/MEG. Epilepsia October 2005;46:1661-1667). (Reprints: Dr M Wolff, University Children's Hospital, Hoppe-Seyler-Str. 1, D-72076 Tuebingen, Germany).

COMMENT. Children with BPE frequently have neuropsychological deficits, and the term "benign" is not always appropriate (Deonna T et al. Dev Med Child Neurol 
2000;42:595-603). A previous report by these authors (Roulet E, Deonna T, Despland PA. Epilepsia 1989;30:564-568) refers to the association of oromotor dyspraxia in a child with benign childhood epilepsy with centrotemporal spikes (BECTS). A fluctuant course of partial seizures involving the face and right arm correlated with the intensity of EEG paroxysms located in the lower rolandic fissure. No structural lesion was detected on MRI (Ped Neur Briefs Nov 1989). A family of 9 children in three generations affected by autosomal dominant BECTS and associated with oral and speech dyspraxia and cognitive impairment is reported from Melbourne, Australia (Scheffer IE et al. Ann Neurol 1995;38:633-642; Ped Neur Briefs Nov 1995). Partial seizures may be repetitive and frequent, more than 100 times daily, as in 2 children with BECTS who showed cognitive decline that improved after seizures resolved (Ong H-T, Wyllie E. Neurology 2000;54:1182-1185; Ped Neur Briefs April 2000;14:28). In one patient the role of antiepileptic medication in the cognitive decline was suspect. A decision to treat BECTS despite invariable spontaneous remission is controversial.

\section{MOVEMENT DISORDERS}

\section{NEURODEVELOPMENTAL OUTCOME OF OPSOCLONUS-ATAXIA}

Cognitive, adaptive behavior, academic, speech and language, and motor abilities were assessed in 19 children with opsoclonus-ataxia and neuroblastoma, followed at University of Southern California, Los Angeles, CA. Patients were examined twice with a minimum interval of 1 year. Age at onset of symptoms ranged from 11 to 31 months (median: 18 months). Age at the time of $1^{\text {st }}$ and $2^{\text {nd }}$ evaluations was a median of 3.1 and 6.4 years, respectively. Cognitive scores improved between testing sessions for the group as a whole, and a larger average gain in IQ/DQ was seen with younger children. Motor abilities improved in gross and fine motor function, but not at an age-appropriate rate. Behavioral and emotional functioning was overall stable and did not worsen significantly with age. Speech and language testing showed persistent deficits in the majority of patients, and all were receiving therapy. The necessity for treatment (steroids or immune-modulating medication) during the interval between evaluations did not influence change in test scores, but a history of relapse was strongly associated with deficits in cognition, academic achievement, and visual motor integration. Of 5 children without relapse, 4 had test scores in the normal range. Despite initial severity of symptoms, a minority of patients had an excellent outcome, with cognitive, academic, behavioral, and motor function within the normal range, and all immunotherapy had been discontinued. Fourteen with multiple relapses, and a chronic course associated with developmental sequelae, required prolonged therapy. (Mitchell WG, Brumm VL, Azen CG et al. Longitudinal neurodevelopmental evaluation of children with opsoclonus-ataxia. Pediatrics October 2005;116:901-907). (Respond: Wendy G Mitchell MD, Neurology Division, Children's Hospital Los Angeles, 4650 Sunset Blvd, Box 82, Los Angeles, CA 90027).

COMMENT. Early and periodic neuropsychological testing is recommended in children diagnosed with opsoclonus-ataxia. Normal cognitive and behavioral development can be expected in the minority of patients without relapse, but a chronic and relapsing course is associated with developmental deficits. This study confirms earlier reports of a 